\title{
UPPER RADICALS AND ESSENTIAL IDEALS
}

\author{
DWIGHT M. OLSON and TERRY L. JENKINS
}

(Received 14 January 1980; revised 23 June 1980)

Communicated by D. E. Taylor

\begin{abstract}
For any class of rings $\mathscr{T}$ it is shown that the class $\mathscr{E}(M)$ of all rings each nonzero homomorphic image of which contains either a nonzero $\mathscr{N}$-ideal or an essential ideal is a radical class. If $\mathscr{N}$ is a class of simple rings the upper radical generated by $\Re, \mathcal{Q}(M)$, is shown to be equal to $\mathcal{E}(\mathscr{R}$ ') where $\mathfrak{T R}^{\prime}$ is the class of simple rings complementary to $\mathfrak{R}$.
\end{abstract}

1980 Mathematics subject classification (Amer. Math. Soc.): 16 A 21.

Key words: upper radical, essential ideal.

The main result in this paper is a new characterization of the upper radical generated by a class of simple rings. We also give some related results for general upper radicals. In Section II we establish some properties of essential ideals, and in Section III prove that if $\pi$ is a class of simple rings the class $\varepsilon(\mathscr{R})$ of all rings $A$ each nonzero homomorphic image of which has either an $\mathscr{T}$-ideal or an essential ideal is a radical class. We also show that $\mathscr{E}(\mathfrak{R})=$ $\mathscr{U}\left(\mathfrak{R}^{\prime}\right)=$ the upper radical determined by the class $\mathfrak{R}^{\prime}$ of simple rings complementary to $\pi$.

The definitions and significant radical theoretic properties used throughout this text may be found in Divinsky (1965) and, in addition, we assume that all rings mentioned are elements of some universal class $\mathscr{Q}$ of associative rings.

OCopyright Australian Mathematical Society 1981 
Recall that an ideal $I$ of a ring $A$ is called essential if $I \neq A$ and $I$ has nonzero intersection with each nonzero ideal of $A$. We can show the following:

LEMMA 1 . If $I$ is a nonzero ideal of $R$ then either $I$ is a direct summand of $R$ or there is an essential ideal of $R$ containing $I$.

Proof. Let $I$ be any nonzero ideal of $R$. Assume $I$ is not a direct summand of $R$. Consider the collection $\Re$ of all ideals which have zero intersection with $I$, (0) $\in M$ so $M \neq \varnothing$ and $M$ is partially ordered by set inclusion. A simple application of Zorn's Lemma gives us a maximal element $M \in \mathfrak{R}$. We shall show that $I+M$ is essential in $R$.

Let $A$ be any nonzero ideal of $R$. If $A \cap I \neq(0)$ then clearly $(I+M) \cap A \neq$ (0). So assume that $A \cap I=(0)$. Then $A \in \mathfrak{R}$. Now, if $A \subseteq M$ we have $(I+M) \cap A \neq(0)$ as desired, so let $A \nsubseteq M$. Then $(A+M) \cap I \neq(0)$ since $A+M$ contains $M$ and $M$ is maximal with respect to $I \cap M=(0)$. Thus there exists $a+m \in A+M$ such that $0 \neq a+m=i \in I$. Then $a=i-m \in I+$ $M$ which implies that $(I+M) \cap A \neq(0)$ unless $a=0$. But if $a=0$ then $m=i$ and $I \cap M \neq(0)$. In any case, we see that $A \cap(I+M) \neq(0)$. Since $I$ is not a direct summand $I+M \neq R$ so $I+M$ is essential in $R$ and $I \subseteq I+M$.

Lemma 2. If $R$ has a homomorphic image with an essential ideal, then $R$ has an essential ideal.

Proof. Let $R / M \neq(0)$ be a homomorphic image of $R$ with essential ideal $K / M$. Then $K / M \neq(0)$ so $M$ is a proper ideal of $K$. Also $K / M \neq R / M$ so $K \neq R$. We shall show that $K$ is essential in $R$.

If $A$ is any nonzero ideal of $R$ then either $A \subseteq K$ or $A \nsubseteq K$. If $A \subseteq K$ then $K \cap A=A \neq(0)$ as desired. On the other hand, if $A \nsubseteq K$ then $A \nsubseteq M$ and we have $(A+M) / M$ a nonzero ideal of $R / M$. Thus $(A+M) / M \cap K / M \neq$ (0), i.e. there exists $a+m \in A+M$ and $k \in K$ such that $[a+m]=[k] \neq[0]$. This implies that $a+m+m_{1}=k+m_{2}$ for some $m_{1}, m_{2} \in M$. But $M \subseteq K$ so $m+m_{1}$ and $k+m_{2} \in K$. Thus $a=\left(k+m_{2}\right)-\left(m+m_{1}\right) \in K$ and we have $a \in A \cap K$. Now if $a=0$ we have $m+m_{1}=k+m_{2}$ which implies $k \in M$. But since $[k] \neq[0]$ this cannot happen. Therefore $A \cap K \neq(0)$ and $K$ is essential in $R$ as desired.

THEOREM 3. The following are equivalent for any ring $R$.

(1) $R$ has no essential ideals. 
(2) Each ideal of $R$ is a direct summand of $R$.

(3) $R$ is a direct sum of simple rings.

Proof. If $R$ has no essential ideals then by Lemma 1 each ideal is a direct summand and hence (1) implies (2). If each ideal in $R$ is a direct summand then clearly it cannot be essential since it has a complementary ideal with zero intersection and so (2) implies (1).

The equivalence of (2) and (3) has been shown by Armendariz (1968) in the case that $R$ is a ring with identity. In general the proof goes as follows:

To show that (3) implies (2) let $R$ be a direct sum of the rings $R_{i}$ ( $i \in I$ ), where each $R_{i}$ is simple. Let $B$ be a proper ideal of $R$. For each subset $J \subseteq I$ let $R_{J}=\Sigma_{j \in J} R_{j}$. Now for any $i, B \cap R_{i}=(0)$ or $R_{i}$. If $B \cap R_{i}=R_{i}$ for all $i$ then $B=R$ which is impossible. Thus there is a $j$ such that $R_{j} \cap B=(0)$. We may use Zorn then to choose a set $J$ maximal with respect to $R_{J} \cap B=(0)$. If $j \in J$ then $R_{j} \subseteq R_{J}+$ $B$. If $i \notin J$ then $R_{J} \subseteq R_{i}+R_{J}$. But the containment is proper so $\left(R_{J}+R_{i}\right) \cap B \neq(0)$ which implies $R_{i} \cap\left(R_{J}+B\right) \neq(0)$. But then $\left(R_{J}+B\right) \cap R_{i}=R_{i}$ since $R_{i}$ is simple. Thus $R_{i} \subseteq R_{J}+B$ and we must have $R=R_{J}+B$ with the sum clearly direct.

The proof that (2) implies (3) has three steps. First, however, we remark:

If each ideal of $R$ is a direct summand of $R$ then we may say the following about any ideal $A$ of $R$.

(1) Any ideal $I$ of $A$ is also an ideal of $R$.

(2) Any ideal $I$ of $A$ is a direct summand of $A$ and also of $R$.

Step (1). Each nonzero ideal of $R$ contains a nonzero simple ideal.

Proof. Let $A \neq(0)$ be an ideal of $R$. If $a \in A$ such that $a \neq 0$ we can use Zorn's Lemma to choose an ideal $T$ of $A$ maximal with respect to $a \notin T$. Then by the remark above $A=T \oplus U$. We show $U$ is simple. For if there is an ideal $V$ of $U$ such that $(0) \neq V$ then, again by the above remark, $U=V \oplus W$ and thus $A=T \oplus V \oplus W$. If $W \neq(0)$, however, $T \oplus W \supsetneq T$ and $T \oplus V \supsetneq T$, so $a \in T \oplus W$ and $a \in T \oplus V$ (by the maximality of $T$ ). Thus in $A=T \oplus V \oplus W$, we have

$$
t+v+0=a=t_{1}+0+w
$$

for some $t, t_{1} \in T, v \in V, w \in W$. Hence $v=0=w$ and $a=t=t_{1}$ $\in T$ which is a contradiction. Thus $W=(0)$ and $V=U$ so $U$ is simple as desired. Note again that this simple $U$ is also an ideal of $R$. 
Step (2). If $S=\sum$ (all simple ideals of $R$ ) then $S=R$.

Proof. If $S \neq R$, then $R=S \oplus K$ with $K \neq(0)$. But by Step (1) we can find a simple ideal of $R$ not contained in $S$.

Step (3). If $R=\Sigma$ (all simple ideals of $R$ ) then $R$ is a direct sum of simple rings.

Proof. Let $\left\{R_{i}\right\}_{i \in I}$ be the collection of simple ideals of $R$ and use Zorn to select $J \subseteq I$ maximal with respect to $\sum_{j \in J} R_{j}$ being a direct sum. Then $R=\oplus \sum_{j \in J} R_{j}$ by Step (1).

\section{III}

Recall that a class $\mathfrak{N}$ of simple rings satisfies property $E$ of Divinsky (1965), p. 5 , and hence we can define an upper radical generated by $\mathfrak{N}$ to be

$\mathscr{U}(\mathfrak{N})=\{A \mid$ each nonzero homomorphic image of $A$ is not in $\mathscr{N}\}$.

We shall give a new characterization of $\mathscr{Q}(\mathscr{T})$.

Let $\mathscr{N}$ be any class of rings. We show that $\mathscr{R}$ determines a radical class as follows.

DEFINITION 4. Let $\mathscr{E}(\mathfrak{R})$ be the class of all rings each nonzero homomorphic image of which contains either a nonzero $\Re$-ideal or an essential ideal.

THEOREM $5 . \mathcal{E}(\mathfrak{T})$ is a radical class.

Proof. (0) qualifies vacuously as a member of $\delta(\mathscr{T})$ and each nonzero homomorphic image of a member of $\mathscr{E}(\mathscr{R})$ is clearly in $\mathscr{E}(\mathscr{R})$ so property ( $R 1)$ is satisfied. Now, let $A \notin \mathcal{E}(\mathfrak{R})$. Then $A$ has a nonzero homomorphic image $A / I$, which has neither a nonzero $\mathfrak{K}$-ideal nor an essential ideal. Let $K / I$ be a nonzero $E(\mathscr{N})$-ideal of $A / I$. By Theorem $3 K / I$ must be a direct summand of $A / I$. Note also that this makes $K / I$ a homomorphic image of $A / I$. Now since $K / I \in \mathcal{E}(\mathfrak{M})$ it must have an essential ideal or a nonzero $\mathscr{N}$-ideal. But if $K / I$ has an essential ideal then Lemma 2 forces $A / I$ to have an essential ideal contrary to the choice of $A / I$. Also if $K / I$ has a nonzero $\mathscr{R}$-ideal then this $\mathfrak{K}$-ideal of $K / I$ is actually an $\Re$-ideal of $A / I$ since $K / I$ is a direct summand of $A / I$. This again is impossible. Thus we have that $A / I$ has no nonzero $\mathscr{E}(\mathfrak{N})$-ideals which is the property $(R 2)$ as desired.

Thus we have that any general class of rings determines a radical class. For a class $\mathfrak{T}$ of simple rings this radical turns out to be well known.

THEOREM 6. For any class $\mathfrak{N}$ of simple rings $\mathscr{U}(\mathfrak{R})=\mathcal{E}\left(\mathfrak{R}^{\prime}\right)$ where $\mathfrak{R}^{\prime}$ is the class of simple rings complementary to $\Re$. 
Proof. Let $A \notin \mathcal{E}\left(\mathfrak{T}^{\prime}\right)$ then $A$ has a nonzero homomorphic image $A / I$ with no essential ideals nor nonzero ideals from $\mathscr{R}$ '. By Theorem $3 A / I$ is then a direct sum of simple rings each of which can be considered an ideal of $A / I$. These cannot be in $\Re^{\prime}$ since $A / I$ has no $\mathscr{T}^{\prime}$-ideals so they are in $\Re$. Each of these summands is a homomorphic image of $A / I$ and thus of $A$, so as a result $A \notin \mathcal{Q}(\mathfrak{R})$. On the other hand if $A \notin \mathcal{U}(\mathfrak{R}), A$ has a nonzero homomorphic image in $\mathscr{N}$ which, since it is simple, clearly has no essential ideals nor $\mathfrak{N}^{\prime}$-ideals. Thus $A \notin \mathscr{E}(\mathfrak{R})^{\prime}$.

Theorems 5 and 6 seem to suggest two problems for further investigation: For a homomorphically closed class of rings $\Re$, what is the relationship between $\mathscr{E}(\mathfrak{R})$ and the lower radical class generated by $\mathfrak{N}$ ? For a class of rings $\mathfrak{K}$ satisfying property $E$ of Divinsky, is there any way to realize $\mathcal{Q}(\mathfrak{T})$ as $\delta(2)$ for some class 2 related to $\mathfrak{R}$ ?

\section{Acknowledgements}

The authors wish to express their gratitude to E. P. Armendariz and the referee for helpful suggestions concerning the proof of Theorem 3.

\section{References}

E. P. Armendariz (1968), 'Direct and subdirect sums of simple rings with unit', Amer. Math. Monthly 75, 746-748.

N. Divinsky (1965), Rings and radicals (University of Toronto Press).

Cameron University

Lawton, OK 73505

USA
University of Wyoming

Laramie, Wyoming 82071

USA 